\title{
Spatial information processing and distortion correction via four-wave mixing
}

\author{
Jeffrey 0 . White \\ Amnon Yariv \\ California Institute of Technology \\ Dept. of Applied Physics \\ Pasadena, California 91125
}

\begin{abstract}
The versatility of degenerate four-wave mixing (D4WM) has made possible its application to two major areas of spatial information processing: distortion correction and mathematical operations. The first area can be divided into predetection and postdetection processing. Distortions can be compensated for before the imaging.process by exploiting the phase-conjugating capability of D4WM. Phase-conjugate mirrors (PCM's) may also find use in optical processors designed to remove distortions after the imaging process is complete and the object is no longer available. Examples of linear operations that can be implemented using the nonlinear optics of D4WM are image convolution and correlation. An example of a nonlinear mathematical operation that can be implemented using D4WM is edge detection of two-dimensional images.
\end{abstract}

Keywords: optical phase conjugation; image processing; distortion correction; four-wave mixing; photolithography; edge enhancement; fiber transmission.

Optical Engineering 21(2), 224-230 (March/April 1982).

\section{CONTENTS}

I. Introduction

II. Distortion correction
A. Predetection
B. Postdetection

III. Mathematical operations

A. Linear operations

B. Edge enhancement

IV. Conclusion

V. References

\section{INTRODUCTION}

The basic D4WM geometry consists of two pairs of coherent counterpropagating beams, all of the same frequency (i.e., degenerate), intersecting inside a medium possessing a third-order optical susceptibility. To get a basic picture of the interaction, one can use coupled mode theory to solve for the evolution of the beam amplitudes, along their directions of propagation. ${ }^{\prime}$ In the simplest case, the amplitudes of the four beams have no temporal variation or spatial variation transverse to the direction of propagation, i.e., the steady-state plane wave case (Fig. 1(a)). In more complicated analyses, the beams may change in time $23 / 8^{6}$ (Fig. 1(b)) or have spatial information on them (Fig. 1(c)), or both. For example, at some initial time, only three input beams may be present, but they stimulate the medium to radiate a fourth output wave. Alternatively, the three input beams may be pulsed, in which case an output will depend on the order,

Invited paper PC-106 received Oct. 1, 1981; accepted for publication Oct. 14, 1981; received by Managing Editor Oct. 29, 1981.

(c) 1982 Society of Photo-Optical Instrumentation Engineers. duration, and separation of the pulses. ${ }^{6}$ Another typical situation is that in which one or both pairs of counterpropagating beams have wavefronts that match both inside and outside the medium, i.e., phase-conjugate pairs (Fig. ld).

In the nonlinear optics (NLO) picture, a nonlinear polarization

$\mathrm{P}=\chi^{(3)} \mathrm{E}_{\mathrm{tot}}{ }^{3}$

is induced in the medium which is proportional to the cube of the total optical field (Fig. 2)

$$
\begin{aligned}
E_{\text {tot }} & =\sum_{i=1}^{4} E_{i}+\text { c.c. } \\
& =\sum_{i=1}^{4} A_{i}\left(\bar{r}_{i}\right) \exp \left(-i\left(\bar{k}_{i} \cdot \bar{r}-\omega t\right)\right)+\text { c.c. } .
\end{aligned}
$$

For simplicity, we assume a single polarization component, and neglect the tensorial aspects of the nonlinear susceptibility $\chi^{(3)}$. In particular, consider the component

$P=\chi^{(3)} E_{1} E_{2} E_{4}^{*}$.

If $E_{1}$ and $E_{2}$ are counterpropagating everywhere inside the medium then, in the presence of $\mathrm{E}_{4}$ (along an arbitrary direction), the polarization

$P=\chi^{(3)} A_{1} A_{2} A_{4}^{*} \exp \left(-i\left(\bar{k}_{4} \cdot \bar{r}-\omega t\right)\right)$ 


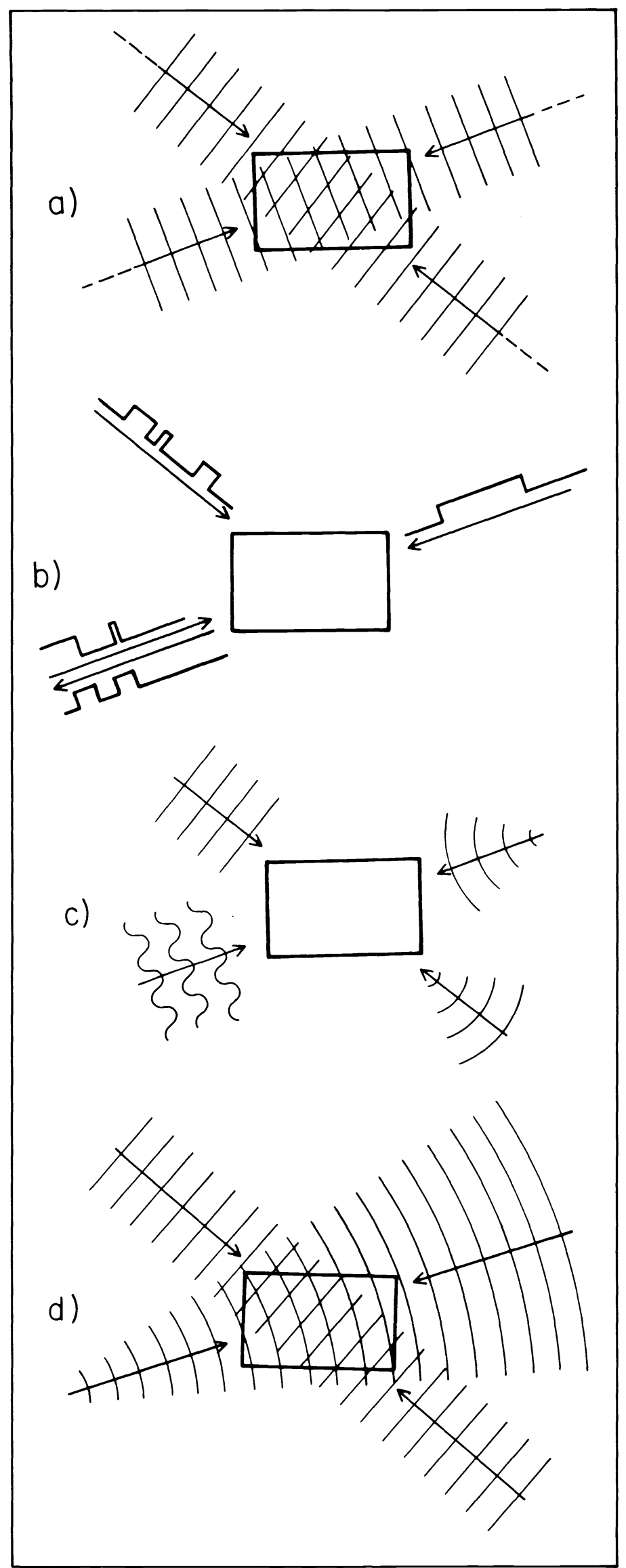

Fig. 1. Examples of D4WM: (a) steady-state plane wave case; (b) pulsed input and output; (c) wavefronts containing spatial information; (d) two phase-conjugate pairs.

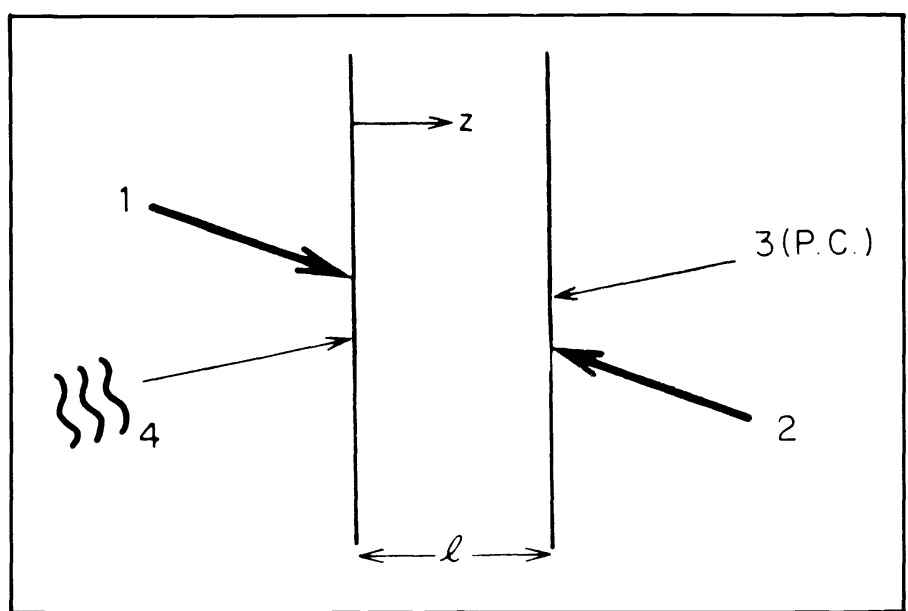

Fig. 2. The four waves included in the total optical electric field.

radiates a wave $A_{3}$, conjugate to $A_{4}$, propagating in the reverse direction with wavevector $\overline{\mathrm{k}}_{3}=-\overline{\mathrm{k}}_{4}$. That is, even if the amplitude $\mathrm{A}_{3}$ is zero at $\mathrm{z}=\ell$, the amplitude will be nonzero at $\mathrm{z}=0$ because the other three waves stimulate the medium to emit $A_{3}$. Thus the combination of a $\chi^{(3)}$ medium pumped by counterpropagating $A_{1}$ and $A_{2}$ constitutes a phase-conjugate mirror. $\mathrm{A}_{4}$ and $\mathrm{A}_{3}$ are, respectively, the incident, input wave and the nonlinear reflection.

In the holographic or grating picture, the optical properties of the medium respond to spatial variations in the intensity of light within the medium. A single beam may have an intensity variation (e.g., a Gaussian profile) in the transverse direction but no phase information is contained in that variation. If we want to record the phase distribution of an object wavefront, it must be interfered with a reference wave, usually planar, inside the medium to write a volume hologram. The object wave can then be reconstructed by illuminating with a plane wave reading beam in the direction of the reference.

In the absence of any fixing process, the hologram will disappear. Uniform illumination will erase holograms in photorefractive crystals; absorption holograms in saturable absorbers will decay according to the lifetime of the metastable state; and media such as $\mathrm{CS}_{2}$ will return to their disordered state by thermal motion.

If, however, we wish to sustain the hologram by the continued writing of the object and reference wave, the reconstruction can be done using a plane wave counterpropagating to the original reference wave, generating the phase conjugate of the object wave.

In D4WM, assigning either a writing or a reading role to any beam is too simple because all combinations of the four beams taken two at a time will interfere to "write" six superimposed holograms. Subject to the Bragg condition, each beam will be diffracted by several of the holograms that are being written by itself and by other beams as well. ${ }^{7}$

The description can be partially sorted out in the plane wave case (Fig. 3). For example, if two plane waves with wavevectors $\mathrm{k}_{1}$ and $\mathrm{k}_{2}$ interfere, the fringes will be sinusoidal, and the hologram reduces to a simple grating characterized by

$\overline{\mathrm{k}}_{\mathrm{g}}=\overline{\mathrm{k}}_{12}= \pm\left(\overline{\mathrm{k}}_{1}-\overline{\mathrm{k}}_{2}\right)$.

This grating can then couple any two waves $\overline{\mathrm{k}}_{\mathrm{i}}, \overline{\mathrm{k}}_{\mathrm{j}}$ satisfying the Bragg condition: $\overline{\mathrm{k}}_{\mathrm{i}}=\overline{\mathrm{k}}_{\mathrm{j}} \pm \overline{\mathrm{k}}_{\mathrm{g}}$.

\section{DISTORTION CORRECTION}

\section{A. Predetection}

A laser beam transmitted from point $A$ to point $B$ through the atmosphere will spread due to the finite size of the beam at $A$. If diffraction were the only factor present, the size of the beam at $B$ would be diffraction limited, and could be made smaller only through the use of a waveguide. Other factors make the diffraction limit difficult to achieve. The beam can be distorted by atmospheric 


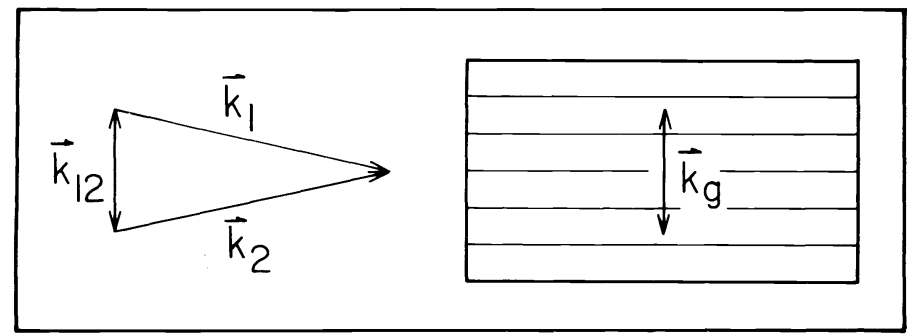

Fig. 3. Two waves interfering to write a grating characterized by $\overline{\mathbf{k}}_{\mathrm{g}}$.

turbulence, or, if the beam is intense enough, it will also encounter, or create, distortions such as thermal blooming. To solve the latter two sources of degradation, we may first transmit a probe beam from $B$ to $A$, so that it acquires or measures the total distortion in transit. Next the phase conjugate is generated at A. Upon reversing its path, the phase-conjugate wave should arrive at $B$ with the same phase and amplitude distribution as the originally transmitted wave. ${ }^{8}$ To correct nonlinear, stationary, lossless distortions perfectly, the phase-conjugate mirror (PCM) must have a reflectivity of unity. ${ }^{9}$ The finite size of the PCM will limit the severity of the distortions which can be corrected.

Imaging an object through a turbulent medium might also be performed using PCM's. ${ }^{10,11}$ Essentially, the distortions can be removed by mixing the distorted object wave with a similarly distorted reference wave. The reference wave originates in the weak reflection of a spotting beam from a small glint or highlight on the object. The reflected light is then amplified and conjugated so that it returns to the region of the glint. The fraction of the light scattered from the glint which is retroreflected will be conjugated again and a suitable iterative system will establish a beam that comes to a focus on the glint, providing a point source reference.

Photolithography by wavefront conjugation has been demonstrated ${ }^{12}$ using a projection system similar to that shown in Fig. 4. A resolution of 800 lines $/ \mathrm{mm}$ over a $6.8 \mathrm{~mm}^{2}$ field and a feature size of $0.75 \mu \mathrm{m}$ were obtained indicating a numerical aperture of 0.48 . The phase-conjugate mirror was a crystal of $\mathrm{LiNbO}_{3}$ pumped by a 413 nm krypton laser. The need for diffraction-limited resolution over a flat field the size of a 3 to 4 in. Si wafer puts very strict limits on the aberrations that can be tolerated in a conventional projection system. Here, however, defects in optical elements, through which the image makes both a forward and a return path, are compensated for by the action of the phase-conjugate mirror. But the action of the PCM depends on the optical quality of the nonlinear medium and the degree to which the pump waves are counterpropagating phase conjugates. $^{12}$

Imaging a three-dimensional object through a fiber is a problem because of modal distortion, differential modal attenuation, and intermode scattering. The first problem can be solved using optical phase conjugation ${ }^{13}$ and has been recently demonstrated using D4WM. ${ }^{14}$ A picture field (see Fig. 5) at the entrance to a fiber excites many real eigenmodes $\mathrm{E}_{\mathrm{mn}}$ :

$f_{o}(x, y, t)=\sum_{m n} A_{m n} E_{m n}(x, y) \exp (i \omega t)$

The phase-velocity dispersion of the fiber modes is responsible for the buildup, over a distance $L$, of phase differences between the modes which smear the image:

$$
f_{1}(x, y, t)=\sum_{m n} A_{m n} E_{m n}(x, y) \exp \left(i\left(\omega t-\beta_{m n} L\right)\right) .
$$

The remedy is to phase conjugate the entire field, midway down the fiber link, in real time by NLO mixing to obtain

$$
f_{2}(x, y, t) \propto \sum_{m n} A_{m n}^{*} E_{m n}(x, y) \exp \left(i\left(\omega t+\beta_{m n} L\right)\right)
$$

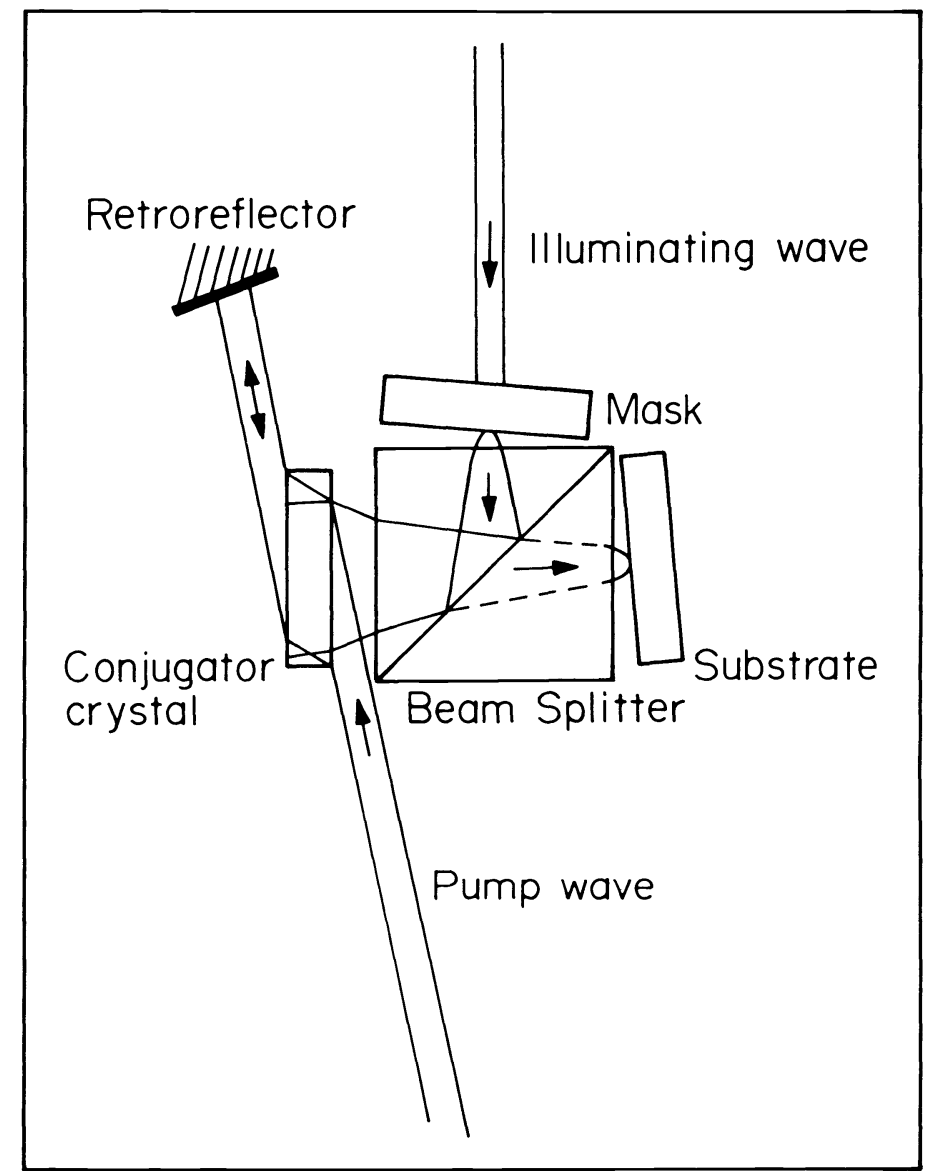

Fig. 4. Schematic of the projection tool used for photolithography. ${ }^{12}$

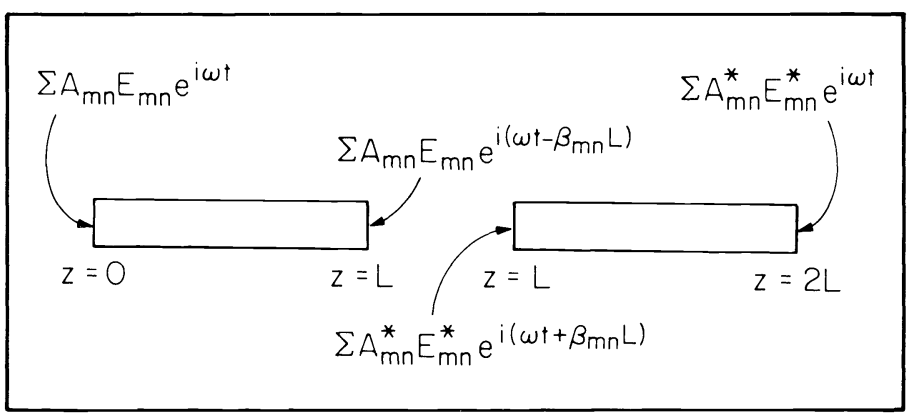

Fig. 5. Compensation (and restoration) for image loss by modal dispersion in a multimode dielectric waveguide using phase conjugation.

If passage through the second half of the fiber produces the same phase changes as the first half, then the field becomes

$f_{3}(x, y, t) \propto \sum_{m n} A_{m n}^{*} E_{m n}(x, y) \exp (i \omega t)$

A second stage of NLO mixing yields a possibly amplified output that is proportional to the original 3-D object field:

$f_{4}(x, y, t) \propto \sum_{m n} A_{m n} E_{m n}(x, y) \exp (i \omega t)$.

\section{B. Postdetection}

Iterative techniques have been proposed for restoring linearly degraded images, ${ }^{15}$ restoring finite energy optical objects from limited spatial and spectral information, ${ }^{16}$ and optically implementing Gersch- 
berg-type algorithms for reconstructing an object from the modulus of its Fourier transform ${ }^{17}$ and image extrapolation. ${ }^{18}$ Coherent optical processors for solving the above restoration problems and processors designed to solve differential and integral equations ${ }^{19,20}$ rely on mirrors to send a wavefront on multiple passes through some kind of filter. It has been proposed that PCM's should find use here because of their wavefront reversal properties and because the possibility of gain should breathe new life into systems which in the past were deemed impractical because of reflection losses. ${ }^{21}$

\section{MATHEMATICAL OPERATIONS}

\section{A. Linear operations}

The usefulness of gratings for optical processing was described in an article of a previous volume of this journal. ${ }^{22}$ For example, a grating placed in the filter plane of a coherent optical processor can translate, add, and subtract input pattern functions (Fig. 6). The amount of translation depends on the period of the grating. If the period is adjusted correctly, two pattern functions may be translated to an overlapping position. Whether they are added or subtracted depends on the phase of the grating. Forming the grating with light, using, say, the photorefractive effect, would offer the advantage of being able to rapidly change the grating in situ. The phase of the grating could be changed by phase modulating one of the writing beams ${ }^{23.24}$ or applying a transverse dc electric field to the medium. ${ }^{25}$ The period of the grating could be adjusted by changing the angle between the writing beams. In the processor of Fig. 6 , intersecting plane waves at the filter plane could be generated from two coherent beams focused on either the input or output plane of the processor. The latter case would correspond to D4WM in the filter plane. Since the directions of the beams carrying the input patterns and the beams writing the grating are traveling in opposite directions, they can be easily separated using a beam splitter. Multiple gratings can also be used to obtain first- or higher-order derivatives of intensity distributions, and restore smeared or multiply exposed images. ${ }^{22}$

The concept can be generalized beyond gratings in the filter plane to the production of more complicated Vander Lugt filters. ${ }^{26}$ In the Vander Lugt technique, a filter or mask is first synthesized by exposing film to the interference pattern of a plane reference wave and the Fourier transform, $\mathrm{H}$, of the desired impulse response, $h$. The developed film is then reinserted into the filter plane, and the object $u$ to be processed is inserted into the input plane. Part of the field leaving the transform plane is proportional to the product $\mathrm{HU}$. Propagating through the second lens gives $h * u$ at the output plane.

As before, a nonlinear medium can be substituted for the film, allowing for rapid modification of the filter. Some caution must be exercised when making such a substitution. Strictly speaking, for an object in the front focal plane of a lens, the exact Fourier transform only exists at one plane in space, the back focal plane. Depending on the confocal parameter of the system, a good or bad approximation to the transform may exist in some noninfinitesimal region about the focal plane. ${ }^{26}$ This puts a limit on the thickness of both volume filters and film emulsions,

$\mathrm{t}<2 \mathrm{f}^{2} \lambda / \mathrm{r}_{\max }{ }^{2}$,

where $r_{\max }$ is the spatial extent of the largest input field, and $f$ is the focal length of the lens.

Also, filters made from photographic film are unaffected by light after the developing process, whereas photorefractive materials remain sensitive to light in the absence of some fixing process. ${ }^{27}$ Birefringent and optically active nonlinear media introduce more complications that can turn out to be useful for improving performance. ${ }^{28}$ Finally, we know that volume gratings have amplitude transmittances that depend strongly on the wavelength and angle of illumination. ${ }^{29}$ So, the synthesis of a dynamic volume filter must be done keeping the intensity, direction, wavelength, and polarization of the readout beam in mind.

For real-time operation of the processor, $h$ and $u$ must be present concurrently. Since they can no longer both be on axis, the filter

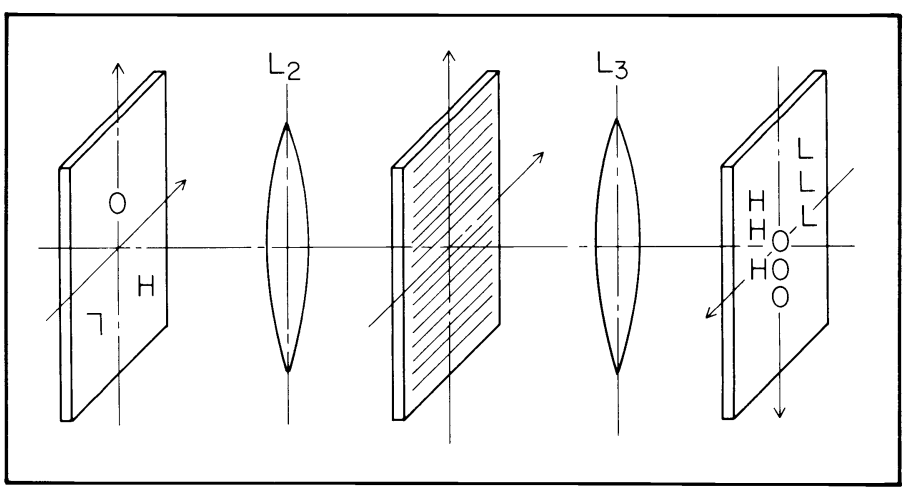

Fig. 6. Optical translation using a grating. (From Ref. 22.)

cannot be read from the same angle at which it was written. For thin nonlinear filters, this is not a problem since the amplitude transmittance does not depend strongly on the angle of illumination. However, the diffraction efficiency will be limited due to the thinness of the filter, and, if a thin saturable absorber is used as the nonlinear medium, the requisite writing intensity may have to be provided by a Q-switched laser. ${ }^{22}$

So, we need to write the filters (gratings) in thick media, subject to the above thickness constraint, but wish to avoid the concomitant angular dependence, particularly on the polar angle relative to the average grating vector.

One approach is to illuminate at the same polar angle, but a different azimuthal angle about the grating vector. ${ }^{30}$ This introduces an astigmatic aberration into the filter output, but satisfactory results were obtained in a system which writes absorption gratings in cryptocyanine dye with $\mathrm{MW} / \mathrm{cm}^{2}$ intensities.

Another approach is to use different wavelengths so that the two input beams can be superimposed. Convolution and correlation can be generated in this way by sum and difference frequency mixing in KDP. ${ }^{31,32}$ No reference is needed but the use of multiple wavelengths (first, second, and third harmonics of Nd:YAG) introduces spatial scaling which may be objectionable. Dispersion in the medium may require precise alignment in order to achieve phase matching.

Another system illuminated both input objects with the $4880 \AA$ wavelength from an argon laser, and used a $6328 \AA$ HeNe beam for the reference. ${ }^{33}$ In contrast to the Vander Lugt sequence, here the two transformed input fields interfere to write a complicated index pattern in a photorefractive medium. Readout is performed by the reference beam, which is almost superimposed with one of the inputs, but misaligned slightly so as to be incident at the Bragg angle. The photorefractive material was $\mathrm{Bi}_{12} \mathrm{SiO}_{20}$ (BSO), which is less sensitive to red than blue, so the reference beam provides a nearly nondestructive readout.

A solution which requires only one wavelength is D4WM. ${ }^{34}$ In the grating picture it corresponds to illumination at the Bragg angle, but in the reverse direction. Again the nonlinear medium is placed in the common focal plane of a two lens system as shown in Fig. 7. Let us examine its operation in the NLO picture, and let all three input fields contain arbitrary amplitude and phase information. The input complex amplitudes $u_{1}(x, y), u_{2}(x, y)$, and $u_{4}(x, y)$, in the outer focal planes are Fourier transformed by propagating to the common focal plane. The transformed fields $\mathrm{U}_{1}, \mathrm{U}_{2}$, and $\mathrm{U}_{4}$ induce nonlinear polarizations in the medium. The polarization

$\mathbf{P}=\chi^{(3)} \mathrm{U}_{1} \mathrm{U}_{2} \mathrm{U}_{4}^{*}$

radiates an output field that propagates essentially backward relative to beam four, returning through lens L. The convolution theorem says that the transform of the product $U_{1} U_{2}$ is the convolution of the transforms $u_{1} * u_{2}$. The definition of correlation is

$\mathrm{u}_{2} \star \mathrm{u}_{4}=\mathrm{u}_{2} * \mathrm{u}_{4}^{*}$ 


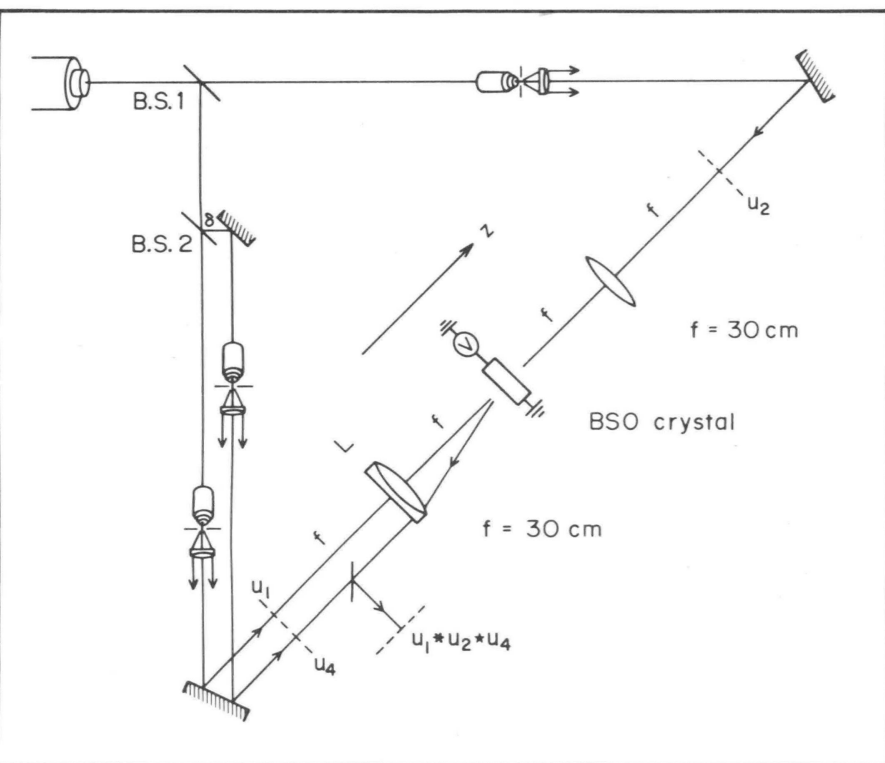

Fig. 7. Experimental apparatus for performing convolution and correlation. Input and output planes shown by dashed lines.

Accordingly, it can be shown that the output field, when evaluated at the plane located a distance $f$ in front of lens $L$, is of the form

$\mathrm{u}_{3} \propto \mathrm{u}_{1} * \mathrm{u}_{2} \star \mathrm{u}_{4}$.

This image processing operation was demonstrated ${ }^{35}$ with the experimental arrangement shown in Fig. 7. The nonlinear medium was a $10 \times 10 \times 3 \mathrm{~mm}^{3}$ crystal of BSO. The application of a transverse ( $\perp$ to $\mathrm{z}) 5$ to $7 \mathrm{kV}$ dc electric field enhances the hologram formed between beams 1 and 4 . The coherence length of the laser must be longer than the greatest optical path length difference, $\delta$, between the beams. The cw argon laser with a power output of $1.6 \mathrm{~W}$ at $5145 \AA$ was initially polarized perpendicular to the plane of the figure.

Two-image processing is performed by simulating a $\delta$ function for the third input, i.e., focusing the third beam at its input plane to give a collimated beam at the transform plane. The interaction is exactly phase matched only when $U_{1}$ and $U_{2}$ are counterpropagating. If, say, $\mathrm{U}_{2}$ is a plane wave, then phase matching (Bragg condition along with conservation of photon energy) will put a constraint on the angular spread, $2 \Delta \theta$, of $\mathrm{U}_{1}$, which we know to be $2 \Delta \theta \sim \Lambda / \mathrm{t}$ for a grating of period $\Lambda$ and thickness t. ${ }^{29}$ The angular spread of $U_{1}$ at the transform plane is related to the spatial extent $d$ of $u_{1}$ at the front focal plane by $2 \Delta \theta=\mathrm{d} / \mathrm{f}$. So, the size of $\mathrm{u}_{1}$ is limited to $\mathrm{d}<\Delta \mathrm{f} / \mathrm{t}$. The average fringe spacing depends on the distance between $\mathrm{u}_{1}$ and $\mathrm{u}_{4}$, which we assume to be $\mathrm{d}$, as follows: $\Lambda=\lambda / 2 \sin \theta \cong \lambda \mathrm{f} / 2 \mathrm{~d}$. So, for this system, the input object size is limited to $3 \mathrm{~mm}$.

$\mathrm{d}<\sqrt{\frac{2 \lambda}{\mathrm{t}}} \mathrm{f}$.

This constraint is seen to be the same as the above constraint on the filter thickness. If $U_{1}$ were a plane wave then the same constraint would apply to the spatial extent of $\mathrm{u}_{2}$.

Typical experimental results are shown in Fig. 8. The first three columns show the input fields $u_{1}, u_{2}$, and $u_{4}$. The fourth column shows the photograph at the output plane. Rows (a) to (c) illustrate correlation, and row (d) illustrates convolution. The output in row (c) clearly demonstrates pattern recognition.

\section{B. Edge enhancement}

The special type of nonlinearity present in the photorefractive effect can be used to advantage in another type of image processing, edge enhancement. ${ }^{36,37}$ To understand how it works, it is necessary to

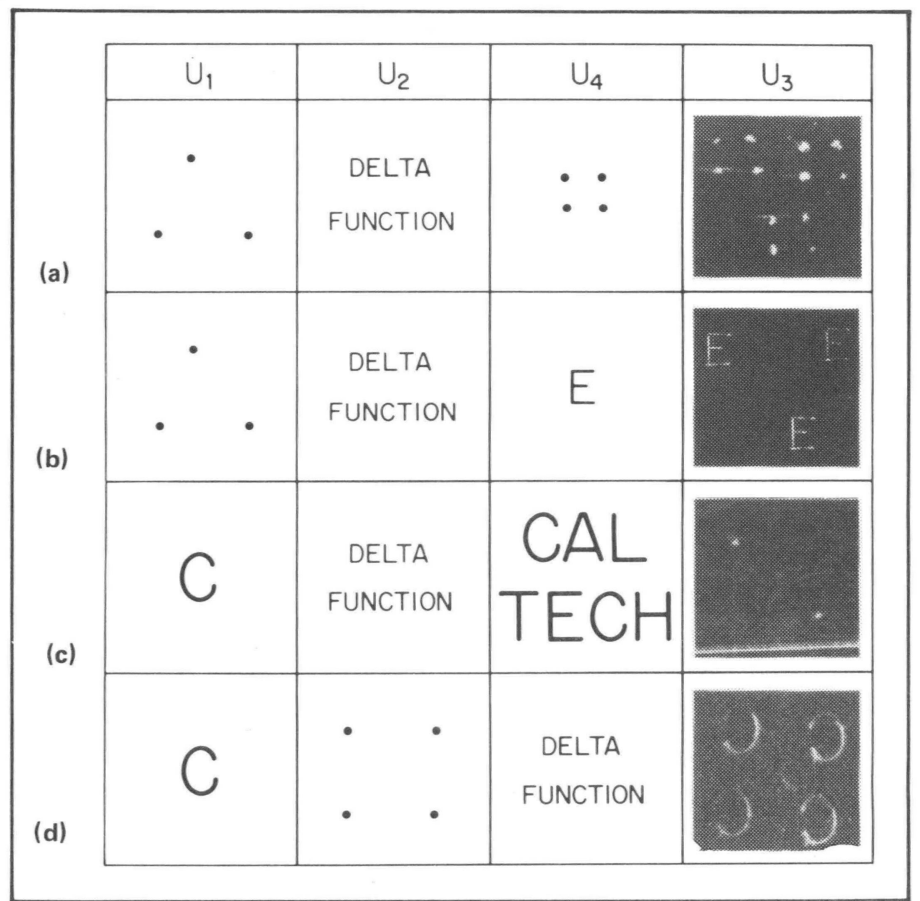

Fig. 8. The first three columns are the input objects $u_{1}, u_{2}$, and $u_{4}$. The last column is photographs of the output $u_{3}$

understand the response of a photorefractive crystal to a spatially varying illumination, e.g.,

$\mathrm{I}(\mathrm{x})=\mathrm{I}_{\mathrm{o}}(1+\mathrm{m} \cos \mathrm{kx})=\mathrm{I}_{\mathrm{o}}+\mathrm{I}_{1} \cos \mathrm{kx}$.

Crystals such as $\mathrm{BSO}, \mathrm{LiNbO}_{3}: \mathrm{Fe}$, and $\mathrm{BaTiO}_{3}$ have localized charge states (traps) in the energy gap between the valence and conduction bands. If incident photons have enough energy, they can excite charges into the conduction band where they diffuse, drift, and are eventually captured by another trap. Since the retrapping is done at random, and the excitation is most probable at the intensity maxima, there is a net migration of charge to the intensity minima (Fig. 9). The density of mobile charges then has a periodic variation whose dc and fundamental components will be

$\rho(\mathrm{x})=\rho_{\mathrm{o}}(1+\mathrm{m} \sin \mathrm{kx})=\rho_{\mathrm{o}}+\rho_{1} \sin \mathrm{kx}$.

Dimensional analysis as well as detailed theoretical and experimental data ${ }^{38}$ concur that for the steady state situation

$\frac{\rho_{1}}{\rho_{\mathrm{o}}} \propto \frac{\mathrm{I}_{1}}{\mathrm{I}_{\mathrm{o}}}=\mathrm{m}$.

The ionized traps will have a similar density. The average charge is zero since the crystal remains neutral, but there is a periodic space charge field $E_{1}$ accompanying the charge separation which modulates the index of refraction via the electro-optic effect. It is this index modulation which constitutes the hologram.

The amplitude of the index modulation is proportional to $E_{1}$, which is proportional to $\mathrm{m}$, so, unlike the optical Kerr effect, an increase in the intensity of one of the optical fields will not necessarily yield a larger effect. The largest index modulation (most efficient hologram) will be produced when two optical fields of the same intensity interfere (Fig. 10).

So, if one wants to enhance the boundary of an object field containing a very intense region and a dark region, choose a reference beam with an intermediate intensity. Image the object onto a photorefractive medium along with the reference. The largest interference fringe modulation will occur at the boundary between the 


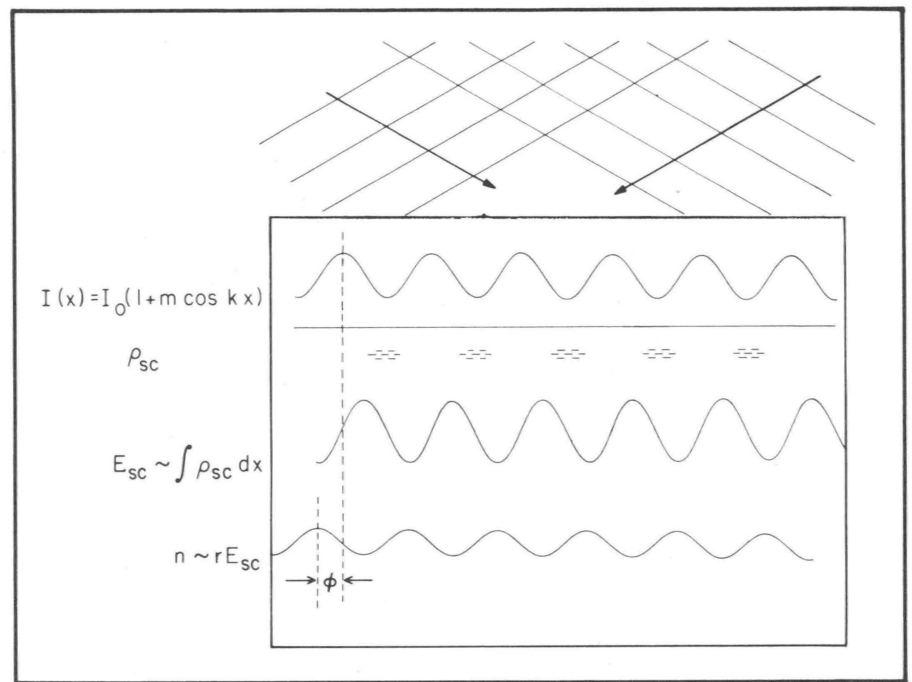

Fig. 9. Light-induced charge migration leading to a change in the index of refraction via the electro-optic effect.

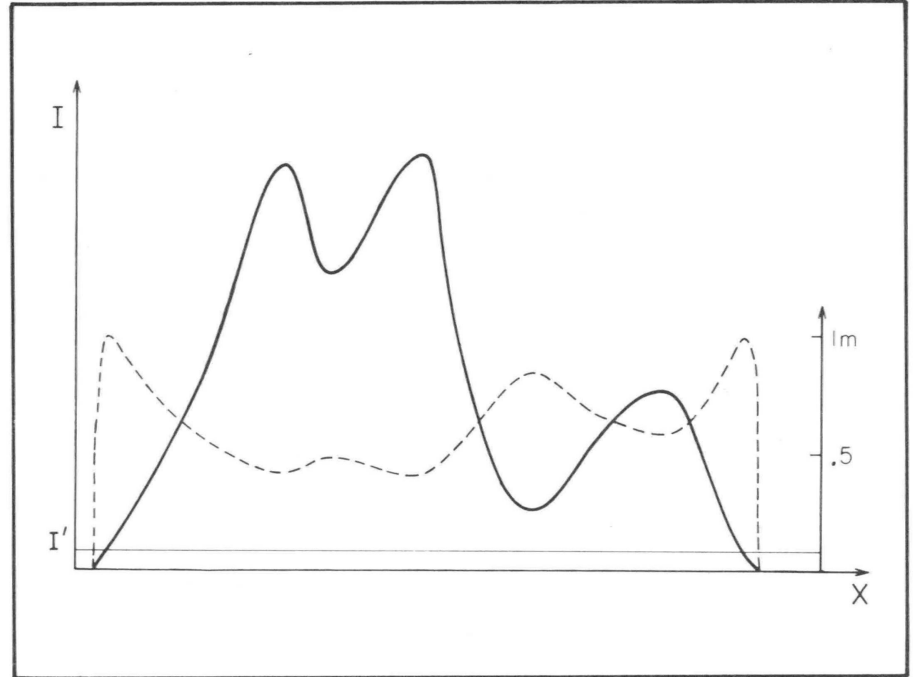

Fig. 10. The modulation index of the fringe pattern formed by the interference of an image having intensity I with a reference beam of uniform intensity $\mathrm{I}^{\prime}$.

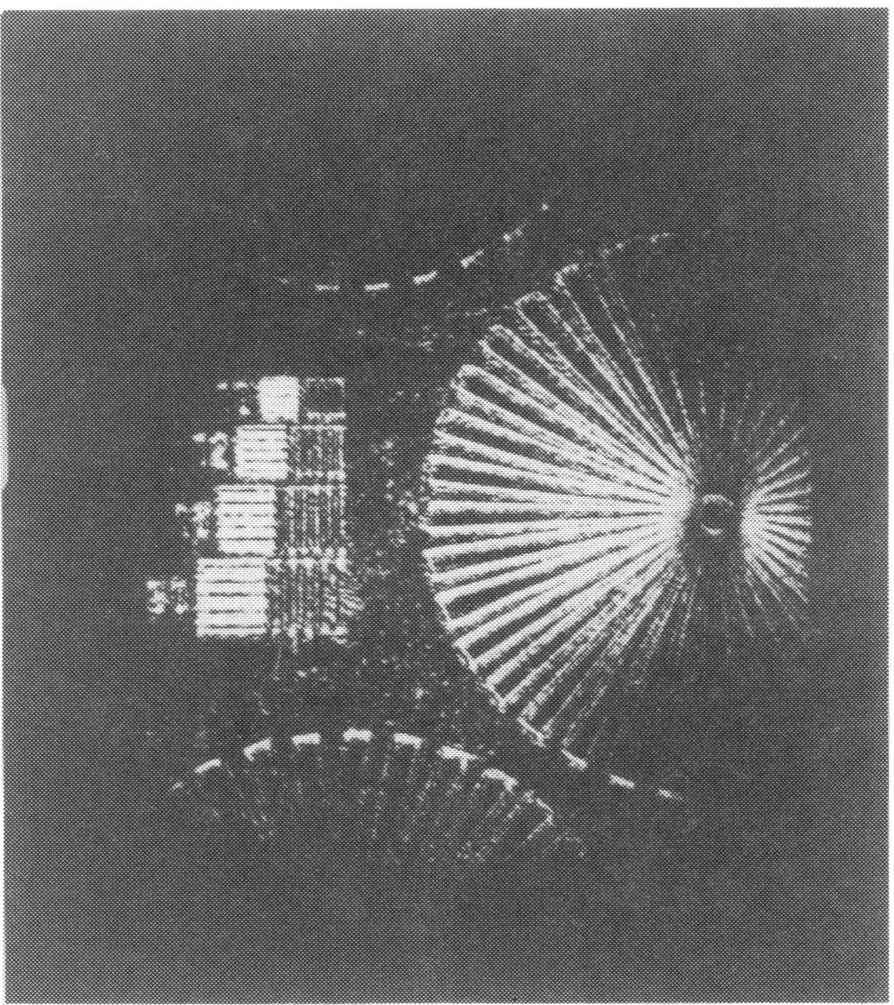

Fig. 11. Images of a resolution chart showing enhancement of only horizontal edges as a result of misaligning the object beam vertically by about $1 \mathrm{~mm}$. The radial pattern is $1 \mathrm{~cm}$ in diameter. The ratio of intensities of the object and reference beams is $1 / 1000$ in the photograph on the left and $1000 / 1$ in the photograph on the right. (From Ref. 37.)

light and dark regions of the image. Now illuminate with a weak third planar wavefront at the Bragg angle, counterpropagating to the reference. The regions of the crystal with the largest index modulation will diffract the most light, yielding an intensity filtering in the image plane (spatial domain). A different type of filtering can also be done by imaging the Fourier transform of the object onto the nonlinear medium. ${ }^{37}$ Horizontal or vertical edges can be selectively enhanced by a slight misalignment of the beams. Some typical experimental results are shown in Fig. 11.

\section{CONCLUSION}

The study of D4WM has yielded i) new understanding about old techniques, e.g., holography and matched filtering;

ii) solutions to problems, e.g., atmospheric distortions and modal distortions in fibers;

iii) new optical elements: the PCM and the dynamic filter; and iv) a common conceptual foundation for widely different optical phenomena, e.g., edge enhancement and phase conjugation.

\section{REFERENCES}

1. A. Yariv and D. M. Pepper, Opt. Lett. 1, 16(1977).

2. R. A. Fisher, B. R. Suydam, and B. J. Feldman, Phys. Rev. A 23, 3071(1981) 
3. D. A. B. Miller, Opt. Lett. 5, 300(1980).

4. T. R. O'Meara, this issue of Opt. Eng. 21(2), 1982.

5. Y. Silverberg and I. Bar-Joseph, IEEE JQE 17, 1967(1981).

6. J. AuYeung and A. Yariv, IEEE JQE 15, 224(1979)

7. B. Fischer, M. Cronin-Golomb, J. O. White, and A. Yariv, Opt. Lett. 6 , (1981).

8. A. Yariv, Opt. Comm. 21, 49(1977).

9. D. M. Pepper and A. Yariv, Opt. Lett. 5, 59(1980).

10. V. Wang, Opt. Eng. 17, 267(1978).

11. T. R. O'Meara, "Applications of Nonlinear Phase Conjugation in Compensated Active Imaging" Proc. of Int'1. Conf. on Lasers '78, Dec. 11-15, 1978.

12. M. D. Levinson, K. M. Johnson, V. C. Hanchett, and K. Chiang, JOSA $71,737(1981)$.

13. A. Yariv, JOSA 66, 301(1976)

14. G. Dunning, private communication.

15. S. Kawata and Y. Ichioka, JOSA 70, 762(1980).

16. H. Stark, D. Cahana, and H. Webb, JOSA 71, 635(1981).

7. J. R. Fienup, Opt. Lett. 3, 27(1978)

18. R. J. Marks II, Appl. Opt. 19, 1670(1980).

19. J. Cederquist and S. H. Lee, JOSA 71, 643(1981).

20. J. Cederquist, JOSA 71, 651(1981).

21. H. H. Szu, "Foundation of Single Frame Image Processing." presented at ICOConference, Optics in Four Dimensions, Ensenada, Mexico, August $4-8,1980$.
22. S. H. Lee, Opt. Eng. 13, 196(1974).

23. H. I. Mandelberg, Opt. Lett. 5, 258(1980).

24. J. P. Huignard and A. Marrakchi, Opt. Comm. 38, 249(1981).

25. N. V. Kukhtarev, V. B. Markov, S. G. Odulov, M. S. Soskin, and V. L. Vinetskii, Ferroelectrics 22, 949(1979).

26. J. W. Goodman, Introduction to Fourier Optics, p. 87 and Ch. 7 , McGraw Hill Book Co., New York (1968).

27. W. D. Burke, D. L. Staebler, W. Phillips, and G. A. Alphonse, Opt. Eng. 17, 308(1978).

28. J. P. Herriau, J. P. Huignard, and P. Auborg, Appl. Opt. 17, 1851(1978).

29. H. Kogelnik, Bell Syst. Tech. J. 48, 2909(1969).

30. B. Carquille and C. Froehly, Appl. Opt. 19, 207(1980)

31. R. A. Eremeeva, V. A. Kudryashov, I. N. Matveev, T. G. Usacheva, and A. I. Chekmenev, Sov. J. Quant. Elect. 5, 1429(1975).

32. R. A. Eremeeva, V. A. Kudryashov, I. N. Matveev, T. G. Usacheva, and A. I. Chekmenev, Sov. J. Quant. Elect. 7, 90(1977).

33. L. Pichon and J. P. Huignard, Opt. Comm. 36, 277(1981).

34. D. M. Pepper, J. AuYeung, D. Fekete, and A. Yariv, Opt. Lett. 3, $7(1978)$.

35. J. White and A. Yariv, Appl. Phys. Lett. 37, 5(1980).

36. J. P. Huignard and J. P. Herriau, Appl. Opt. 17, 2671(1978)

37. J. Feinberg, Opt. Lett. 5, 330(1980).

38. J. P. Huignard, J. P. Herriau, P. Auborg, and E. Spitz, Opt. Lett. 4. 21(1979). 